Article

\title{
Simultaneous Determination and Quantification of Triterpene Saponins from Camellia sinensis Seeds Using UPLC-PDA-QTOF-MS/MS
}

\author{
Xuejin $\mathrm{Wu}^{\dagger}{ }^{+}$, Lingyan Jia ${ }^{\dagger}$, Jiafan Wu, Yawen Liu, Hyunuk Kang, Xiaobo Liu, Pan Li, Puming He, \\ Youying $\mathrm{Tu}$ * and Bo $\mathrm{Li}$ * \\ Department of Tea Science, Zhejiang University, 866 Yuhangtang Road, Hangzhou 310058, China; \\ wuxuejin@zju.edu.cn (X.W.); 11416013@zju.edu.cn (L.J.); 21716168@zju.edu.cn (J.W.); 21816164@zju.edu.cn (Y.L.); \\ 11616111@zju.edu.cn (H.K.); 11616053@zju.edu.cn (X.L.); 21716067@zju.edu.cn (P.L.); pmhe@zju.edu.cn (P.H.) \\ * Correspondence: youytu@zju.edu.cn (Y.T.); drlib@zju.edu.cn (B.L.); Tel.: +86-571-88982743 (Y.T. \& B.L.) \\ + These authors contributed equally to this work.
}

Received: 6 September 2019; Accepted: 20 October 2019; Published: 22 October 2019

\begin{abstract}
Saponins in the Camellia sinensis seeds have a broad spectrum of biological properties and application potentials. However, up to now, no chromatographic methods have been developed to provide full fingerprinting and quality assurance for these saponins. This research aimed to develop a novel method to tentatively identify and quantify saponins in $C$. sinensis seeds by ultra-high-performance liquid chromatography coupled with photo-diode array detector and quadrupole time-of-flight mass spectrometry (UPLC-PDA-QTOF-MS/MS), and compare it with the classic vanillin-sulfuric acid assay. Fifty-one triterpene saponins, including six potentially new compounds, were simultaneously detected by UPLC-PDA-MS/MS, and their chemical structures were speculated according to the retention behavior and fragmentation pattern. The total saponin content in the crude extract and the purified saponin fraction of $C$. sinensis seeds were quantified to be $19.57 \pm 0.05 \%$ ( $\mathrm{wt} \%$ ) and $41.68 \pm 0.09 \%$ ( $\mathrm{wt} \%$ ) respectively by UPLC-PDA at $210 \mathrm{~nm}$, while the corresponding values were determined to be $43.11 \pm 3.17 \%$ (wt \%) and $56.60 \pm 5.79 \%$ (wt \%) respectively by the vanillin-sulfuric acid assay. The developed UPLC-PDA -MS/MS method could determine specified saponins, and is more reliable for quantifying the $C$. sinensis seed saponins than the classic spectrophotometric method. It is of great significance for the future investigations and applications of these saponins.
\end{abstract}

Keywords: saponin; Camellia sinensis seed; UPLC-PDA-QTOF-MS/MS; identification; quantitation; vanillin-sulfuric acid

\section{Introduction}

Camellia sinensis (L.) O. Kuntze, belonging to the family of Theaceae, is a woody perennial tree with great economic importance [1]. Tea manufactured from the leaves of $C$. sinensis is second in popularity only to water as a beverage in the world, and possesses many benefits to human health [2]. In addition to tea leaves, the seeds of $C$. sinensis contain a large number of bioactive constituents including saponins, flavonoids, unsaturated fatty acids, and polysaccharides, and are considered as a valuable resource for food, agriculture, pharmaceuticals, aquaculture, and the cosmetic industry [3-7].

Oleanane-type triterpene saponins were determined as one of the major bioactive ingredients in C. sinensis seeds. So far, more than 50 saponin compounds have been isolated and identified from the seeds of C. sinensis cultivated in China, Japan, Sri Lanka, and India [8]. Pharmacological studies indicated that these saponins could protect gastric mucosa of rats induced by ethanol or indomethacin [9], inhibit gastric emptying and alcohol absorption in mice [10], suppress ascites cancer S180 cells, myelocytic 
leukemia K562 cells, promyelocytic leukemia HL-60 cells, cisplatin-resistant ovarian cancer OVCAR-3 and A2780/CP70 cells in vitro and in vivo [11,12], block inflammatory pathways composed of AKT, IKK, and NF- $\mathrm{KB}$ in macrophages stimulated by fluorescein isothiocyanate-dextran, sodium nitroprusside and lipopolysaccharide [13], and have a wide spectrum of anti-bacterial and anti-fungal activities [14-16]. In addition, $C$. sinensis seed saponins could promote uptake of $\mathrm{Cd}$ by Amaranthus caudatus, and were more efficient than ethylenediaminetetracetic acid (EDTA) [17].

Although $C$. sinensis seed saponins have gotten increasing attention due to a broad spectrum of biological properties and application potentials, the studies on the analysis methods for these compounds are still limited. These saponins occur as a multicomponent mixture with similar structures and polarities, which causes a challenge for their isolation and determination, and thus limits in-depth investigation into their bioactivities and applications. Spectrophotometric and liquid chromatographic methods are usually employed to determine saponins from various plant materials [18]. The vanillin-sulfuric acid assay is the most commonly selected spectrophotometric method for saponin quantification since it is simple, fast and inexpensive to operate $[19,20]$. However, this method can only measure the total saponin content, and its color reaction is not specific [21]. Hyphenated techniques coupling thin-layer chromatography (TLC), high-performance liquid chromatography (HPLC) and ultra-performance liquid chromatography (UPLC) with an ultraviolet detector (UV), evaporative light scattering detector (ELSD) and mass spectrometry (MS) have been applied to characterize and quantify plant saponins [22-26]. Nevertheless, no chromatographic methods have been developed to provide full fingerprinting and quality assurance for $C$. sinensis seed saponins up to now. The major difficulties are providing effective separation and identification without enough saponin standards.

The aim of the present study was to develop an effective ultra-high-performance liquid chromatography coupled with photo-diode array detector and mass spectrometry (UPLC-PDA-MS/MS) method for the simultaneous detection and quantification of $C$. sinensis seed saponins. Compound identification was performed based on the MS/MS data, predication of polarity and structure information of all reported saponins from the seeds of the genus Camellia. In addition, this chromatographic method and vanillin-sulfuric acid assay were compared to evaluate their reliability for quantifying C. sinensis seed saponins.

\section{Results and Discussion}

\subsection{Optimization of UPLC-PDA-QTOF-MS Chromatography}

Ultra-high-performance liquid chromatography coupled with photo-diode array detector and quadrupole time-of-flight mass spectrometry (UPLC-PDA-QTOF-MS) was used to detect the saponins in the crude extract and total saponin fraction of $C$. sinensis seeds in this work. The crude extract was obtained under reflux with $70 \%$ methanol at $70{ }^{\circ} \mathrm{C}$, and then was purified by successive extraction with different polar solvents (petroleum ether, ethyl acetate, and 1-butanol) and D101 column chromatography to yield the total saponins. This procedure is one of the classical methods for the extraction and purification of Camellia seed saponins as reviewed by Guo et al. recently [8]. The chromatographic conditions were optimized to achieve effective separation, symmetric peak shape and short run time. Three chromatographic columns, namely the Welch Ultimate UHPLC XB-C 18 $(1.8 \mu \mathrm{m}, 100 \mathrm{~mm} \times 2.1 \mathrm{~mm}$ i.d. $)$, Waters ACQUITY UPLC BEH C $18(1.7 \mu \mathrm{m}, 100 \mathrm{~mm} \times 2.1 \mathrm{~mm}$ i.d.) and Waters ACQUITY UPLC HSS T3 column $(1.8 \mu \mathrm{m}, 150 \mathrm{~mm} \times 2.1 \mathrm{~mm}$ i.d.) were pretested, and the best separation efficiency was obtained with the HSS T3 column. Acetonitrile was selected as the mobile phase due to its much lower absorption at lower UV wavelengths, improved separation, and reduced column back pressure compared with methanol [27]. The addition of formic acid in the mobile phase improved the peak shape, sensitivity, and retention time of individual saponins, which was consistent with the previous report [28]. Under the developed chromatographic condition, 51 saponins were simultaneously detected from the crude extract of $C$. sinensis seeds $(2.0 \mathrm{mg} / \mathrm{mL})$ in negative ion mode (Figure 1A), and 40 corresponding peaks were obviously observed under a PDA detector at a 
wavelength of $210 \mathrm{~nm}$ (Figure 1B). For the purified total saponin fraction $(2.0 \mathrm{mg} / \mathrm{mL})$, the UPLC/MS total ion chromatogram was similar to that of the crude extract (data not shown), and 50 saponins were detected at $210 \mathrm{~nm}$ (Figure 1C). The majority of saponins of $C$. sinensis seeds possess no chromophores, and could be detected at the non-specific ultraviolet wavelengths around $210 \mathrm{~nm}$. This structure feature led to lower detection sensitivity under the ultraviolet (UV) detector compared with MS, and explained that some minor peaks were not observed in the UV chromatogram of the samples. For the purified total saponin fraction, the peaks from the UV and MS total ion current chromatograms were almost all corresponding with each other except for peak 3, indicating UV detector is more applicable for detecting $C$. sinensis seed saponins at higher concentrations.

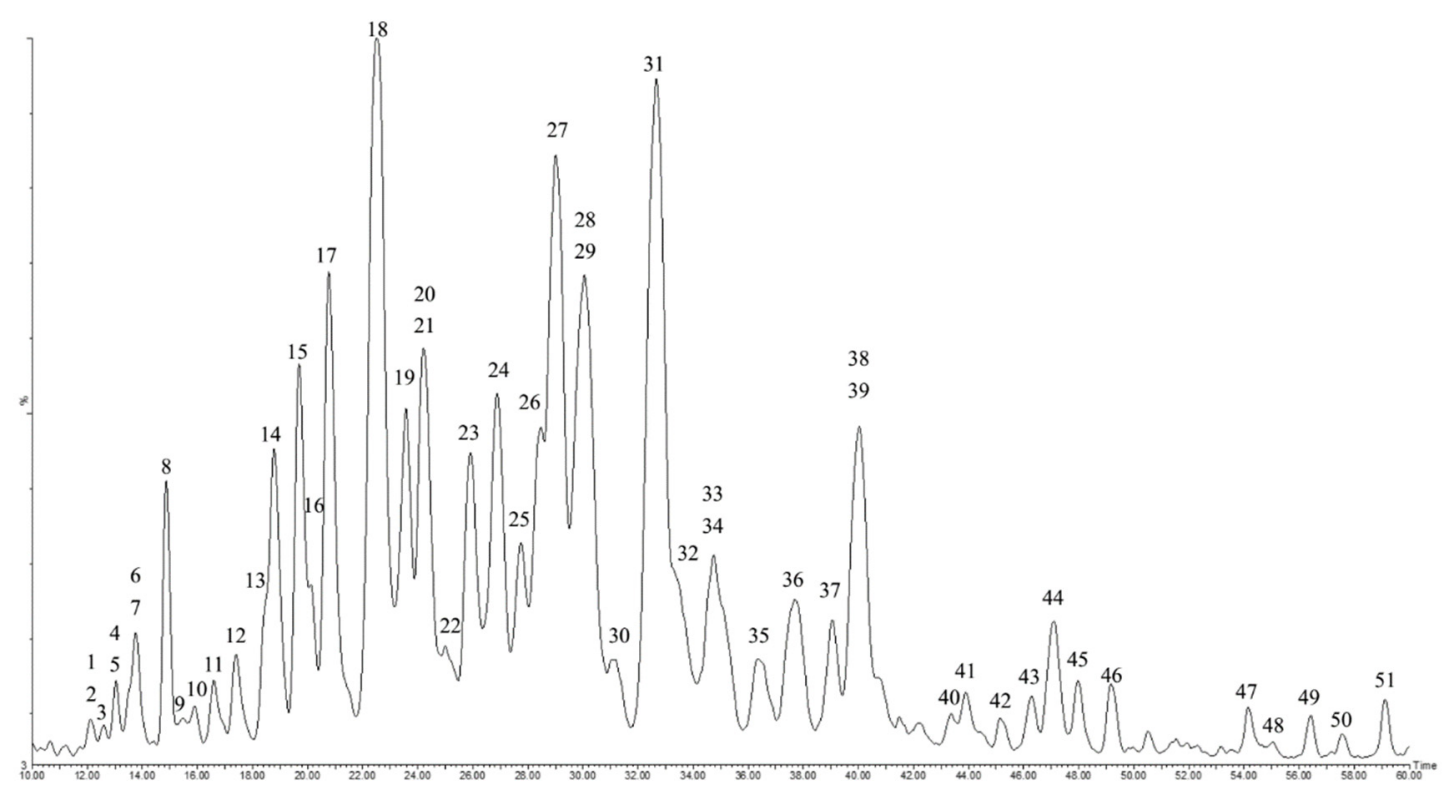

(A)

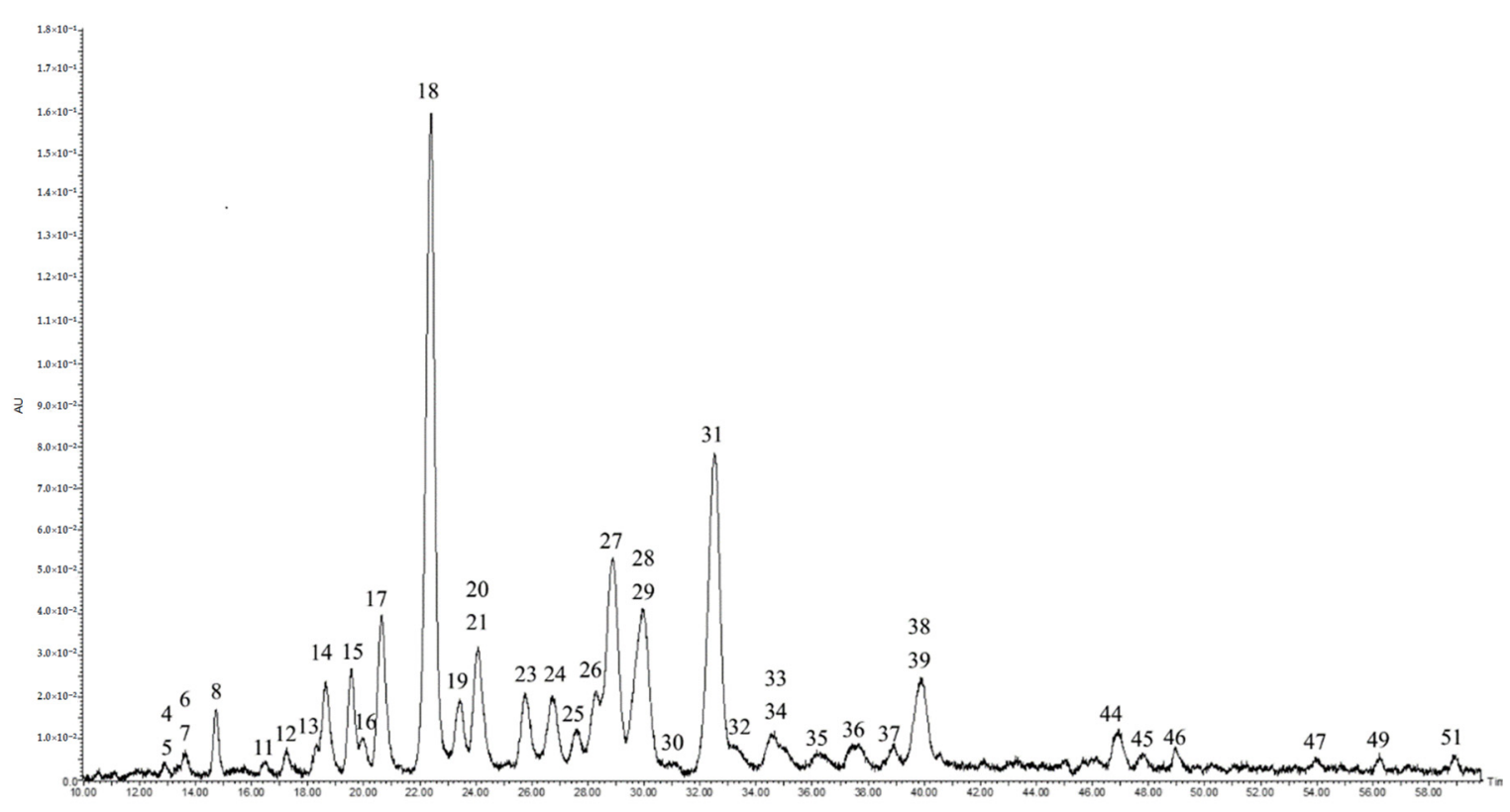

(B)

Figure 1. Cont. 


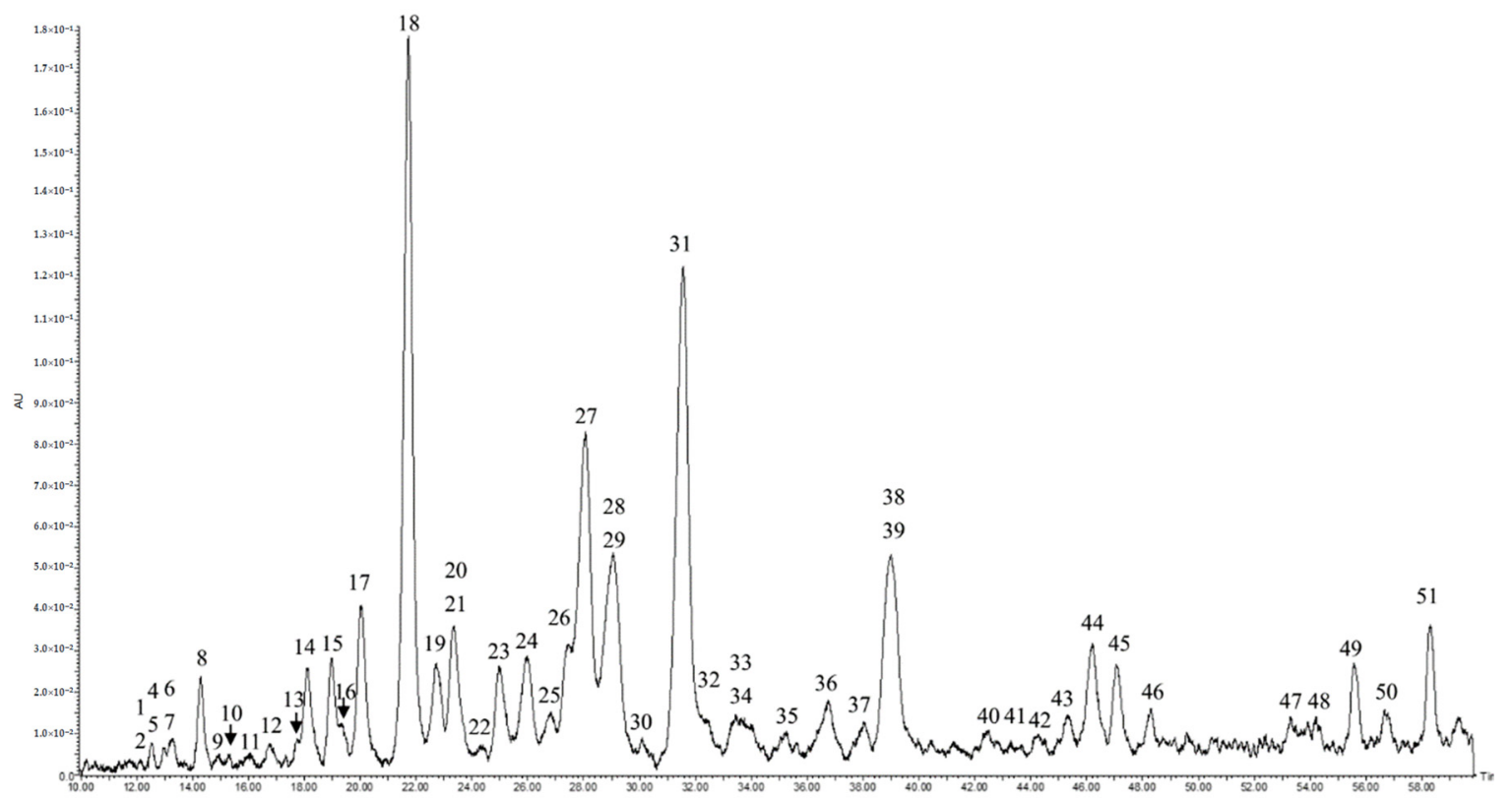

(C)

Figure 1. Saponins from the seeds of Camellia sinensis detected by ultra-high-performance liquid chromatography coupled with photo-diode array detector and quadrupole time-of-flight mass spectrometry (UPLC-PDA-QTOF-MS). (A) UPLC/MS total ion chromatogram of the crude extract of C. sinensis seeds $(2.0 \mathrm{mg} / \mathrm{mL})$ in negative ion mode. (B) UPLC-PDA chromatogram of the crude extract of C. sinensis seeds $(2.0 \mathrm{mg} / \mathrm{mL})$ at $210 \mathrm{~nm}$. (C) UPLC-PDA chromatogram of the total saponin fraction of C. sinensis seeds $(2.0 \mathrm{mg} / \mathrm{mL})$ at $210 \mathrm{~nm}$.

\subsection{Compound Characterization of Saponins by UPLC-QTOF-MS/MS}

In our system, the signal intensity of the saponins obtained in the negative ionization mode was superior to that in the positive ionization mode, which was consistent with the previous studies [24]. In the precursor ion full-scan spectra, the 51 saponin peaks were detected with parent ions $[\mathrm{M}-\mathrm{H}]^{-}$at m/z 1157.5841-1315.6046 (Table 1). Among these compounds, eleven peaks have unique molecular ions $[\mathrm{M}-\mathrm{H}]^{-}$at $m / z 1157.5841$ (peak 38), $\mathrm{m} / \mathrm{z} 1185.5774$ (peak 45), $\mathrm{m} / \mathrm{z} 1189.5742$ (peak 5), $\mathrm{m} / \mathrm{z} 1215.5892$ (peak 37), $m / z 1219.5854$ (peak 3), $m / z 1243.5835$ (peak 46), m/z 1257.5991 (peak 43), m/z 1269.6005 (peak 51), $\mathrm{m} / \mathrm{z} 1299.6105$ (peak 50), $\mathrm{m} / \mathrm{z} 1303.6111$ (peak 21), and $\mathrm{m} / \mathrm{z} 1315.6111$ (peak 42), respectively. In addition, thirteen groups with similar molecular weights were found at $\mathrm{m} / \mathrm{z} 1171.5630-1171.5670$ (peaks 7, 28), m/z 1173.5783-1173.5809 (peaks 15, 19), m/z 1187.5587-1187.5953 (peaks 4, 8, 36, 40), m/z 1201.5759-1201.5779 (peaks 24, 32), m/z 1217.5686-1217.5693 (peaks 1, 6, 10), m/z 1229.5699-1229.5725 (peaks 2, 13, 18, 27, 31), m/z 1231.5838-1231.5873 (peaks 11, 14, 22), m/z 1259.5807-1259.5844 (peaks 12, 16, 20, 29, 33), m/z 1261.5947-1261.5989 (peaks 9, 25), m/z 1271.5805-1271.6146 (peaks 30, 39, 49), $m / z$ 1273.5938-1273.5993 (peaks 17, 23, 34, 41), $\mathrm{m} / \mathrm{z} 1285.5945-1285.5987$ (peaks 44, 47), and $\mathrm{m} / \mathrm{z}$ 1301.5922-1301.5942 (peaks 26, 35, 48). 
Table 1. Saponins detected in the crude extract of $C$. sinensis seeds by UPLC-PDA-QTOF-MS/MS.

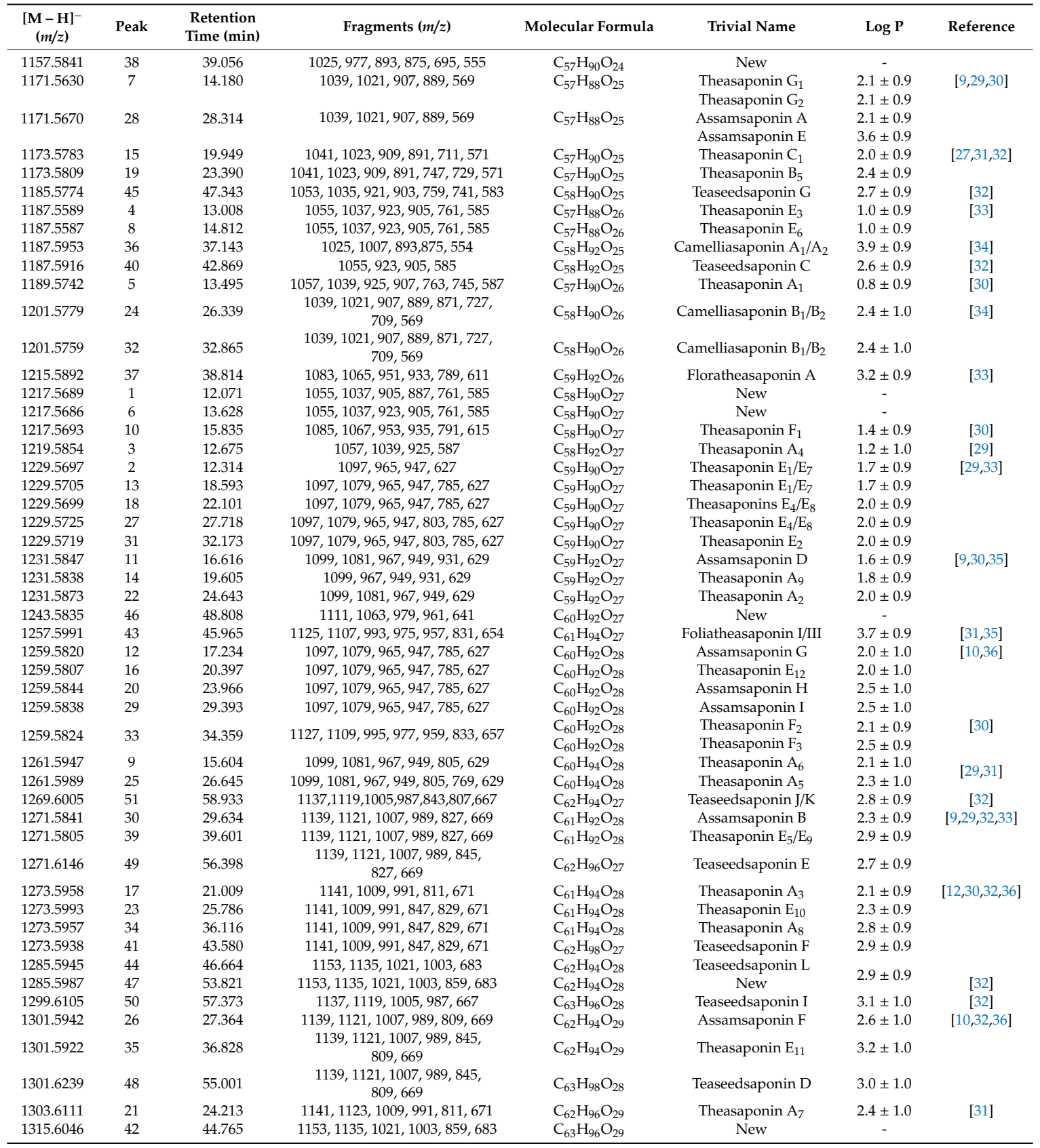

Further identification was conducted based on the MS/MS spectra and fragmentation pathways, referring to previous reports on the saponins isolated from C. sinensis seeds [8]. First of all, the eleven peaks with unique ion $[\mathrm{M}-\mathrm{H}]^{-}$were tentatively identified. Peak 3 has the parent ion $[\mathrm{M}-\mathrm{H}]^{-}$at $m / z 1219.5854$ and four main fragment ions at $m / z 1057(-162 \mathrm{Da}), 1039(-162-18 \mathrm{Da})$, $925(-162-132 \mathrm{Da})$ and $587(-162-132-162-176 \mathrm{Da})$. The information was consistent with the structure of theasaponin $\mathrm{A}_{4}$ (21-O-angeloyl-theasapogenol A 3-O- $\beta$-D-galactopyranosyl- $(1 \rightarrow 2)-[\beta$-Dglucopyranosyl-( $1 \rightarrow 2)$ - $\alpha$-L-arabinopyranosyl-( $1 \rightarrow 3)$ ]- $\beta$-D-glucuronopyranoside) [29], which has the corresponding fragments including $[\mathrm{M}-\mathrm{H}-\text { glucopyranosyl }]^{-}$, $\left[\mathrm{M}-\mathrm{H}-\right.$ glucopyranosyl $-\mathrm{H}_{2} \mathrm{O}^{-}$, [M - H - glucopyranosyl - arabinopyranosyl $]^{-}$and [M - H - glucopyranosyl - arabinopyranosyl galactopyranosyl - glucuronopyranosyl] ${ }^{-}$. In the same way, the peaks 5, 21, 37, 43, 45, 50, and 51 were tentatively identified as theasaponin $A_{1}$ [30], theasaponin $A_{7}$ [31], floratheasaponin $A$ [33], foliatheasaponin I/III [31,35], teaseedsaponin $G$, teaseedsaponin I, and teaseedsaponin $\mathrm{J} / \mathrm{K}$ [32], 
respectively. In addition, the peak 18 was identified as theasaponin $E_{1}$ by comparing the retention time and MS/MS spectra with standard theasaponin $\mathrm{E}_{1}$.

No saponins with the same molecular weight as the peak 38, 42 and 46 have been reported. Their possible structures were try to speculated based on the known saponins from the seeds of the genus Camellia. The peak 38 has parent ion $[\mathrm{M}-\mathrm{H}]^{-}$at $\mathrm{m} / \mathrm{z} 1157.5841$, and fragment ions at $m / z 1025$ (-132 Da), 977 (-162 - $18 \mathrm{Da}), 893$ (-132 - $132 \mathrm{Da}), 875$ (-132 - $132-18 \mathrm{Da})$, and 555 $(-132-132-162-176 \mathrm{Da})$. These data suggested that the molecular weight of its sapogenin was around 556, and the sugar moiety contained two pentoses, one hexose, and one hexosuronic acid. Among all the reported saponins from the Camellia seeds, only the sapogenin of oleiferasaponin $D_{1}$ in the seeds of Camellia oleifera [37], namely 22-O-angeloyl-camelliagenin A, has the corresponding molecular weight. The other structure possibilities of the sapogenin results from the angeloyl-linked positions at C-16 and C-28, and the tigloyl is another possible acyl group. The MS/MS fragmentation pattern of peak 38 were consistent with the sugar chain of 38 known saponins from $C$. sinensis seeds, including theasaponin $A_{1}-A_{3}[30], A_{8}, A_{9}[12], B_{5}$ [31], $C_{1}$ [29], $E_{1}-E_{10}[26,29,33,36], F_{1}-F_{3}[30], G_{1}$, $\mathrm{H}_{1}$ [29], $\mathrm{G}_{2}$ [36], assamsaponin A-E [9], teaseedsaponin C, E-G, J-L [35], and floratheasaponin A, I, III $[31,33,35]$, and the oligosaccharidic moiety of all the reported Camellia seed saponins is located at $\mathrm{C}-3$. So the compound 38 was tentatively identified as 16/22/28-O-angeloyl/tigloyl-camelliagenin A 3 -O- $\beta$-D-galactopyranosyl- $(1 \rightarrow 2)$-[ $\beta$-D-xylopyranosyl- $(1 \rightarrow 2)-\alpha$-L-arabinopyranosyl-( $1 \rightarrow 3)]-\beta$-Dglucuronopyranosyl. The peak 42 showed parent ion $[\mathrm{M}-\mathrm{H}]^{-}$at $\mathrm{m} / \mathrm{z} 1315.6046$, and the fragment ions at $m / z 1153(-162 \mathrm{Da}), 1135$ (-162 - 18 Da), 1021 (-162 - $132 \mathrm{Da}), 1003(-162-132-18 \mathrm{Da}), 859$ (-162 - 132 $162 \mathrm{Da})$, and $683(-162-132-162-176 \mathrm{Da})$. Its sapogenin may be the similar with that of teaseedsaponin $\mathrm{L}$, namely 16, 22-di-O-acetyl-21-O-hexenoyl-theasapogenol E [35]. The hexenoyl usually occurs at C-21 or C-22 of the known Camellia seed saponins, and the acetyl group may link to the hydroxyl at positions $16,21,22$, and 28 . Its sugar chain possibly corresponded to $-\beta$-D-galactopyranosyl- $(1 \rightarrow 2)-[\beta$-Dglucopyranosyl-(1 $\rightarrow 2)-\alpha$-L-arabinopyranosyl-( $(1 \rightarrow 3)]-\beta$-D-glucuronopyranosyl, which was the oligosaccharidic moiety of theasaponin $\mathrm{A}_{4}-\mathrm{A}_{7}$ [31], $\mathrm{E}_{11}, \mathrm{E}_{12}$ [36], assamsaponin F-I [10], teaseedsaponin A, B, D, H, I [32], and camelliasaponin $B_{1}, C_{1}$ [33] in C. sinensis seeds. The peak 46 has parent ion $[\mathrm{M}-\mathrm{H}]^{-}$at $\mathrm{m} / \mathrm{z}$ 1243.5835, which corresponded to the sapogenin of teaseedsaponin $\mathrm{H}, 21-\mathrm{O}$-hexenoyl-28-O-acetyl-theasapogenol E [32]. Other structure possibilities of its sapogenin result from the positions of hexenoyl and acetyl as peak 42 . The fragment ions occurred at $m / z 1111(-132 \mathrm{Da}), 979(-132-132 \mathrm{Da}), 961(-132-132-18 \mathrm{Da})$, and $641(-132-$ $132-162-176 \mathrm{Da})$, suggesting its sugar chain probably was $-\beta$-D-galactopyranosyl- $(1 \rightarrow 2)-[\beta-\mathrm{D}-$ xylopyranosyl-( $(1 \rightarrow 2)-\alpha$-L-arabinopyranosyl- $(1 \rightarrow 3)]-\beta$-D-glucuronopyranosyl as peak 38 . The speculated chemical structures of compounds $38,42,46$ and another three unknown saponins $(1,6,44$, or 47) mentioned were shown in Figure 2. The six compounds were firstly found in the C. sinensis seeds, and their structures should be further confirmed by NMR spectra in the future work. 


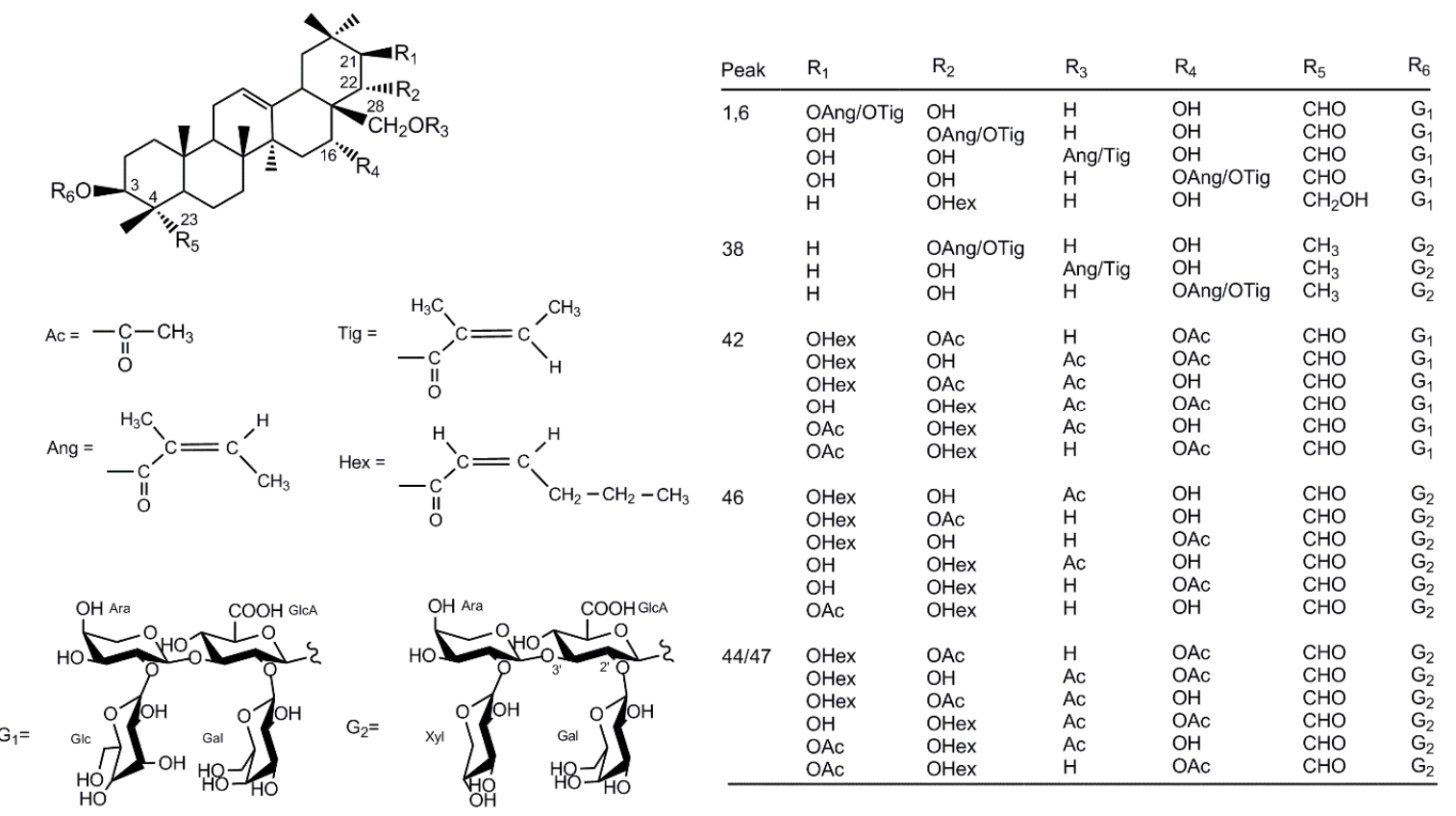

Figure 2. Possible chemical structures of the six potentially new saponins from the Camellia sinensis seeds. Ac $=$ acetyl, Ang = angeloyl, Tig = tigloyl, Hex = hexenoyl, Ara = arabinopyranosyl, $\mathrm{Gal}=$ galactopyranosyl, Glc $=$ glucopyranosyl, GlcA = glucuronopyranosyl, $\mathrm{Xyl}=$ xylopyranosyl .

For five groups with ion $[\mathrm{M}-\mathrm{H}]^{-}$at $\mathrm{m} / \mathrm{z} 1173.5783-1173.5809$ (peaks 15, 19), $\mathrm{m} / \mathrm{z} 1231.5838-1231.5873$ (peaks 11, 14, 22), m/z 1261.5947-1261.5989 (peaks 9, 25), m/z 1273.5938-1273.5993 (peaks 17, 23, 32, 41), and $m / z$ 1301.5922-1301.6239 (peaks 26, 35, 48), the amount of compounds in each group was equal to the number of known $C$. sinensis seed saponins with the similar molecular weight and corresponding fragments. In order to predict the elution order of isomers in each group, Log P value was calculated in SciFinder by launching a chemical structure from ChemBioDraw Ultra 14. Log P is defined as a logarithm of the distribution coefficient of the compound in the liquid/liquid extraction system of water and $n$-octanol. It provides direct information on hydrophobicity, and is frequently used to predict water solubility, soil (sediment)/water partition coefficient, absorption, distribution, metabolism, excretion, and toxicity (ADMET). The higher the Log P value, the more the compound is hydrophobic [38,39]. The group composed of peaks 15 and 19 was taken as an example to elucidate the identification of saponins with a similar molecular weight. The two peaks showed almost the same pseudomolecular ion $[\mathrm{M}-\mathrm{H}]^{-}$at $m / z 1173.58$, and similar fragment ions at $m / z 1041, \mathrm{~m} / z 1023$, $m / z 909, m / z$ 891, $m / z 747, m / z 729, m / z 711$, and $m / z 571$, which were consistent with theasaponin $C_{1}$ [29] and theasaponin $B_{5}$ [31]. The two compounds may have different sapogenins and the same oligosaccharidic moiety, and are undistinguishable from each other by MS/MS spectra. The Log $\mathrm{P}$ values of theasaponin $C_{1}$ and $B_{5}$ were estimated to be $2.0 \pm 0.9$ and $2.4 \pm 0.9$, respectively, suggesting that the former saponin was more hydrophilic, and should be eluted earlier than the latter one. So the peak 15 was tentatively determined as theasaponin $C_{1}$, and the peak 19 was tentatively determined as theasaponin $B_{5}$. Similarly, another four groups of isomers with ion $[\mathrm{M}-\mathrm{H}]^{-}$at $\mathrm{m} / \mathrm{z} 1231.5838-1231.5873$, $\mathrm{m} / \mathrm{z}$ 1261.5947-1261.5989, $\mathrm{m} / \mathrm{z}$ 1273.5938-1273.5993, and $\mathrm{m} / \mathrm{z}$ 1301.5922-1301.6239 were tentatively identified as assamsaponin $\mathrm{D}$ (peak 11) [9], theasaponin $\mathrm{A}_{9}$ (peak 14) [12], theasaponin $\mathrm{A}_{2}$ (peak 22) [30], theasaponin $A_{6}$ (peak 9) [31], theasaponin $A_{5}$ (peak 25) [29], theasaponin $A_{3}$ (peak 17) [30], theasaponin $E_{10}$ (peak 23) [36], theasaponin $A_{8}$ (peak 32) [12], teaseedsaponin $F$ (peak 41) [32], assamsaponin $\mathrm{F}$ (peak 26) [10], teaseedsaponin D (peak 35), [35] and theasaponin $\mathrm{E}_{11}$ (peak 48) [36].

For the other eight groups with ion $[\mathrm{M}-\mathrm{H}]^{-}$at $\mathrm{m} / \mathrm{z} 1171.5630-1171.5670$ (peaks 7, 28), $\mathrm{m} / \mathrm{z}$ 1187.5587-1187.5953 (peaks 4, 8, 36, 40), m/z 1201.5759-1201.5779 (peaks 24, 33), m/z 1217.5686-1217.5693 (peaks 1, 6, 10), m/z 1229.5699-1229.5725 (peaks 2, 13, 18, 27, 31), m/z 1259.5807-1259.5844 (peaks 12, $16,20,29,34$ ), m/z 1271.5805-1271.6146 (peaks 30, 39, 49), and m/z 1285.5945-1285.5987 (peaks 44, 47), 
the amount of compounds in each group did not match to the number of known C. sinensis seed saponins with the corresponding structures, and/or the $\log \mathrm{P}$ values of some isomers were the same. So saponins in the eight groups could only be partly identified according to the MS/MS spectra and the Log P values. The groups with ion $[\mathrm{M}-\mathrm{H}]^{-}$at $m / z$ 1187.5587-1187.5953 (peaks 4, 8, 36, 40), $m / z$ 1217.5686-1217.5693 (peaks 1, 6, 10) and $m / z$ 1285.5945-1285.5987 (peaks 44, 47) were discussed here as examples to illustrate the identification of these saponins.

For the group with $[\mathrm{M}-\mathrm{H}]^{-}$at $m / z$ 1187.5587-1187.5953 (peaks 4, 8, 36, 40), the three peaks 4,8 and 40 had the similar fragments at $m / z 1055(-132 \mathrm{Da}), m / z 1037(-132-18 \mathrm{Da}), m / z 923(-132$ - $132 \mathrm{Da}), m / z$ 905(-132 - 132- $18 \mathrm{Da}), m / z$ 761(-132 - 132 - $162 \mathrm{Da}), m / z 585$ (-132 - 132 - 162 $176 \mathrm{Da})$. These data were consistent with the structures of theasaponin $\mathrm{E}_{3}$, theasaponin $\mathrm{E}_{6}$ [33] and teaseedsaponin $C$ [32] isolated from $C$. sinensis seeds, which had the corresponding fragments including $[\mathrm{M}-\mathrm{H}-\text { arabinopyranosyl }]^{-},\left[\mathrm{M}-\mathrm{H}-\text { arabinopyranosyl }-\mathrm{H}_{2} \mathrm{O}\right]^{-},[\mathrm{M}-\mathrm{H}-$ arabinopyranosyl xylopyranosyl $]^{-}$, [M - $\mathrm{H}$ - arabinopyranosyl - xylopyranosyl $\left.-\mathrm{H}_{2} \mathrm{O}\right]^{-}$, [M - $\mathrm{H}$ - arabinopyranosyl xylopyranosyl - galactopyranosyl $]^{-}$and [M - H - arabinopyranosyl - xylopyranosyl - galactopyranosyl - glucuronopyranosyl] $]^{-}$. The calculated molecular weight of teaseedsaponin $\mathrm{C}$ is 0.043 higher than that of theasaponin $\mathrm{E}_{3}$ and theasaponin $\mathrm{E}_{6}$. In addition, the $\log \mathrm{P}$ value of theasaponin $\mathrm{E}_{3}$ and $\mathrm{E}_{6}$ were both 1.0, while the value of teaseedsaponin $C$ was 2.6. So the Peak 4 and 8 were tentatively determined as either theasaponin $E_{3}$ or $E_{6}$, and the peak 40 was teaseedsaponin $C$. The peak 36 possesses fragment ions at m/z 1025 (-162 Da), m/z 1007 (-162 - 18 Da), m/z 893 (-162 - 132 Da), m/z 875(-162 - 132 $18 \mathrm{Da})$ and $\mathrm{m} / \mathrm{z} 554(-162-132-162-176 \mathrm{Da})$, suggesting its sapogenin and sugar chain are different from that of peaks 4,8 and 40 . This compound has not been found in $C$. sinensis seeds before, and its mass spectrometry data is in accordance with camelliasaponin $A_{1}$ and $A_{2}$ from C. japonica seeds [34].

Peak 1, 6 and 10 yielded pseudo-molecular ion $[\mathrm{M}-\mathrm{H}]^{-}$at $m / z$ 1217.5686-1217.5693. Theasaponin $\mathrm{F}_{1}$ is the unique known saponin with the corresponding molecular weight from $C$. sinensis seeds [30]. The peak 10 had fragment ions at $m / z$ 1085, m/z 1067, m/z 953, m/z 935, m/z 791 and m/z 615, which was corresponded to the fragments of theasaponin $\mathrm{F}_{1}$ including $[\mathrm{M}-\mathrm{H}-\mathrm{xylopyranosyl}]^{-}$, $\left[\mathrm{M}-\mathrm{H} \text { - xylopyranosyl }-\mathrm{H}_{2} \mathrm{O}\right]^{-},[\mathrm{M}-\mathrm{H} \text {-xylopyranosyl-arabinopyranosyl }]^{-},\left[\mathrm{M}-\mathrm{H}-x_{\text {xylopyranosyl }}\right.$ - arabinopyranosyl $\left.-\mathrm{H}_{2} \mathrm{O}\right]^{-},[\mathrm{M}-\mathrm{H} \text { - xylopyranosyl - arabinopyranosyl - galactopyranosyl }]^{-}$and [M - H - xylopyranosyl - arabinopyranosyl - galactopyranosyl - glucuronopyranosyl $]^{-}$, respectively. The peaks 1 and 6 showed the fragment ions at m/z 1055 (-162 Da), m/z $1037(-162-18 \mathrm{Da}), m / z$ $923(-162$ - $132 \mathrm{Da}), m / z 905(-162-132-18 \mathrm{Da}), m / z 887(-162-132-18-18 \mathrm{Da}), m / z 761$ $(-162-132-162 \mathrm{Da})$, and $m / z 585(-162-132-162-176 \mathrm{Da})$. The information indicated that the sapogenins of peak 1 and 6 might be similar with that of theasaponin $E_{3}, E_{6}$, and teaseedsaponin $C$, namely 21-O-angeloyl-theasapogenol $\mathrm{E}$ and 22-O-hexenoyl-theasapogenol $\mathrm{C}$ [32,35]. Other structure probabilities of the sapogenins resulted from the presence of angeloyl and tigloyl located at C-16, C-21, $\mathrm{C}-22$, and C-28 of theasapogenol E. There was much chance that their oligosaccharidic moiety were - $\beta$-D-galactopyranosyl-( $1 \rightarrow 2)$-[ $\beta$-D-glucopyranosyl- $(1 \rightarrow 2)$ - $\alpha$-L-arabinopyranosyl- $(1 \rightarrow 3)]-\beta$-Dglucuronopyranosyl as that of Compound 42 (Figure 2). The two compounds 1 and 6 were firstly found in $C$. sinensis seeds.

Peak 44 and 47 have the pseudo-molecular ion $[\mathrm{M}-\mathrm{H}]^{-}$at $\mathrm{m} / \mathrm{z} 1285.5945$ and $\mathrm{m} / \mathrm{z}$ 1285.5987, and the fragment ions at $m / z 1153$ (-132 Da), $m / z 1135$ (-132 - $18 \mathrm{Da}), m / z$ 1021(-132 - 132 $\mathrm{Da}), m / z 1003(-132$ - 132 - $18 \mathrm{Da}), m / z 859(-132-132-162 \mathrm{Da})$, and $m / z 683(-132-132$ - 162 - $176 \mathrm{Da})$. Among all the reported C. sinensis seed saponins, only teaseedsaponin L corresponds to these data [35]. So either of the peaks 44 and 47 might be teaseedsaponin $\mathrm{L}$, and the another one (probably unreported) in the seeds of $C$. sinensis. Compared with teaseedsaponin $\mathrm{L}$, the potentially-new one might have the diverse substitutions including hexenoyl and acetyl at C-16, C-21, C-22, and C-28 of the sapogenin, and possess the same oligosaccharidic moiety, $-\beta$-D-galactopyranosyl- $(1 \rightarrow 2)-[\beta-\mathrm{D}$-xylopyranosyl- $(1 \rightarrow 2)-\alpha$-L-arabinopyranosyl- $(1 \rightarrow 3)]-\beta-\mathrm{D}-$ glucuronopyranosyl (Figure 2). 
In the same way, the other five groups with ion $[\mathrm{M}-\mathrm{H}]^{-}$at $m / z 1171.5630-1171.5670, m / z$ 1201.5759-1201.5779, $\mathrm{m} / \mathrm{z}$ 1229.5699-1229.5725, $\mathrm{m} / \mathrm{z}$ 1259.5807-1259.5844, and $\mathrm{m} / \mathrm{z} 1271.5805-1271.6146$ were identified referencing the reported saponins isolated from $C$. sinensis seeds. The retention time, MS/MS data, and Log P values of saponins detected in C. sinensis seeds are summarized in Table 1. It was noticed that the elution order of the 51 peaks were not all in the sequence of computer-calculated Log P values. Although Log P is widely used in many quantitative structure-activity relationship (QSAR) models to estimate critical physico-chemical properties of compounds, most of these models exhibit only modest predictive ability due to the chemical diversity of the systems modeled [40]. In this work, Log P value only was used as a reference basis to predict the elution order of isomers. However, it should be noticed that these speculated results need to be further verified due to the limitation of Log $\mathrm{P}$ value and the different conformation of isomers which can influence the retention time. Completely-accurate identification results should be obtained depending on the availability of all appropriate standards, or development of efficient on-line hyphenated techniques coupling separation and structural identification. The liquid chromatography (LC)-solid-phase extraction (SPE)-nuclear magnetic resonance (NMR)-mass spectrometry (MS) coupling can greatly simplify the structure identification of unknown compounds in the complex mixtures, without the need for purifying of the analytes [41]. This technique has become a valuable tool for pharmaceutical and natural product research, and is worth being investigated for the analysis of $C$. sinensis seed saponins in the future. Although this UPLC-QTOF-MS/MS method could only provide inference of the compound structures, it was the first time to establish a liquid chromatography method for simultaneously detecting 51 saponins from the $C$. sinensis seeds.

\subsection{Quantitation of Saponins by UPLC-PDA}

Although several chromatographic techniques have been developed for saponin analysis, LC with UV detection remains the routine method for the quality control of saponin in herbal products [18]. In this work, the saponins in $C$. sinensis seeds were quantified by their response to PDA, and theasaponin $E_{1}$ was used as a standard. The calibration curves were linear over the concentration range of $0-1000 \mu \mathrm{g} / \mathrm{mL}$ for theasaponin $\mathrm{E}_{1}$. The linear regression equation was $\mathrm{Y}=8233.9 \mathrm{X}+43043$, where $X$ is the concentration and $Y$ is the peak area. The $95 \%$ confidence intervals for the regression slope and y-intercept were calculated to range between 8032.5-8435.3 and -62879.8-148965.6. The high correlation coefficient value $\left(R^{2}=0.9994\right)$ indicated a good correlation between standard concentrations and their peak areas within the test ranges. The LLOD $(S / N=3)$ and LLOQ $(S / N=10)$ of theasaponin $\mathrm{E}_{1}$ were $0.0313 \mu \mathrm{g} / \mathrm{mL}$ and $0.1043 \mu \mathrm{g} / \mathrm{mL}$, respectively. The intra- and inter-day of accuracy and precision data at three concentrations of theasaponin $E_{1}$ were summarized in Table 2. The accuracy for theasaponin $E_{1}$ ranged from $-0.092 \%$ to $0.079 \%$, and the precision was between $0.013 \%$ and $0.152 \%$. These results met the Guidance for Industry: Bioanalytical Method Validation (2013) proffered by the US Food and Drug Administration (FDA), which recommended the mean value should be within 15\% of the nominal value for accuracy, and the precision should not exceed $15 \%$ of coefficient of variation (CV). The developed UPLC-PDA method was applied to analyze 70\% methanol extract and the total saponin fraction of $C$. sinensis seeds. The individual saponins were quantified, and the total saponin content of the crude extract and the total saponin fraction were calculated to be $19.57 \pm 0.05 \%$ (wt \%) and $41.68 \pm 0.09 \%$ (wt \%), respectively (Table 3 ). The disadvantage of this method was that all saponin compounds were quantified by the same one standard substance, not by each their own standards. This is due to no commercial C. sinensis seed saponin compounds are available, and purify of all these saponins as standards is still difficult so far. In view of the structure similarity of these compounds, this UPLC-PDA method is still relative reliable for quantitation of $C$. sinensis seed saponins. 
Table 2. Intra-day and inter-day accuracy and precision of theasaponin $\mathrm{E}_{1}$ at three concentrations.

\begin{tabular}{|c|c|c|c|c|c|c|c|c|}
\hline \multirow{2}{*}{$\begin{array}{c}\text { Nominal } \\
\text { Concentration } \\
(\mu \mathrm{g} / \mathrm{mL})\end{array}$} & \multicolumn{2}{|c|}{$\begin{array}{c}\text { Calculated } \\
\text { Concentration } \\
(\mu \mathrm{g} / \mathrm{mL})\end{array}$} & \multicolumn{2}{|c|}{ Accuracy (DEV, \%) } & \multicolumn{2}{|c|}{ Precision (RSD, \%) } & \multicolumn{2}{|c|}{ 95\% Confidence Interval } \\
\hline & Intra-Day & Inter-Day & Intra-Day & Inter-Day & Intra-Day & Inter-Day & Intra-Day & Inter-Day \\
\hline 60 & $\begin{array}{c}59.952 \pm \\
0.091\end{array}$ & $\begin{array}{c}59.945 \pm \\
0.012\end{array}$ & 0.080 & 0.092 & 0.152 & 0.020 & $59.726-60.178$ & 59.915-59.975 \\
\hline 100 & $\begin{array}{c}100.079 \pm \\
0.095\end{array}$ & $\begin{array}{c}100.046 \pm \\
0.056\end{array}$ & 0.079 & 0.046 & 0.095 & 0.056 & $99.843-100.315$ & 99.907-100.185 \\
\hline 500 & $\begin{array}{c}499.960 \pm \\
0.065\end{array}$ & $\begin{array}{c}500.013 \pm \\
0.091\end{array}$ & 0.008 & 0.003 & 0.013 & 0.018 & 499.799-500.122 & $499.787-500.239$ \\
\hline
\end{tabular}

Table 3. Saponin content of the crude extract and total saponin fraction of $C$. sinensis seeds determined by UPLC-PDA.

\begin{tabular}{|c|c|c|c|c|}
\hline \multirow{2}{*}{ Peak } & \multicolumn{2}{|c|}{ Crude Extract } & \multicolumn{2}{|c|}{ Total Saponin Fraction } \\
\hline & Retention Time (min) & Content (wt \%) & Retention Time (min) & Content (wt \%) \\
\hline $\begin{array}{l}1 \\
2\end{array}$ & ND & ND & 12.12 & $0.08 \pm 0.01$ \\
\hline 3 & ND & ND & ND & ND \\
\hline $\begin{array}{l}4 \\
5\end{array}$ & 12.95 & $0.13 \pm 0.01$ & 12.59 & $0.31 \pm 0.01$ \\
\hline 6 & 13.26 & $0.04 \pm 0.01$ & 12.88 & $0.14 \pm 0.01$ \\
\hline 7 & 13.72 & $0.15 \pm 0.01$ & 13.29 & $0.46 \pm 0.01$ \\
\hline 8 & 14.76 & $0.49 \pm 0.01$ & 14.31 & $0.99 \pm 0.01$ \\
\hline 9 & ND & ND & 14.90 & $0.15 \pm 0.01$ \\
\hline 10 & 15.77 & $0.11 \pm 0.02$ & 15.34 & $0.16 \pm 0.01$ \\
\hline 11 & 16.55 & $0.14 \pm 0.01$ & 16.08 & $0.17 \pm 0.01$ \\
\hline 12 & 17.32 & $0.17 \pm 0.01$ & 16.76 & $0.32 \pm 0.01$ \\
\hline 13 & 18.34 & $0.16 \pm 0.01$ & 17.77 & $0.26 \pm 0.02$ \\
\hline 14 & 18.72 & $0.83 \pm 0.01$ & 18.17 & $1.04 \pm 0.02$ \\
\hline 15 & 19.60 & $1.02 \pm 0.01$ & 19.00 & $1.11 \pm 0.02$ \\
\hline 16 & 19.99 & $0.34 \pm 0.01$ & 19.39 & $0.44 \pm 0.02$ \\
\hline 17 & 20.72 & $1.07 \pm 0.01$ & 20.01 & $1.60 \pm 0.01$ \\
\hline 18 & 22.43 & $5.43 \pm 0.02$ & 21.82 & $7.62 \pm 0.02$ \\
\hline 19 & 23.42 & $0.31 \pm 0.01$ & 22.77 & $0.82 \pm 0.02$ \\
\hline $\begin{array}{l}20 \\
21\end{array}$ & 23.95 & $0.76 \pm 0.01$ & 23.32 & $1.14 \pm 0.02$ \\
\hline 22 & ND & ND & 24.33 & $0.15 \pm 0.01$ \\
\hline 23 & 25.81 & $0.48 \pm 0.01$ & 24.97 & $0.96 \pm 0.02$ \\
\hline 24 & 26.80 & $0.42 \pm 0.02$ & 25.99 & $0.98 \pm 0.01$ \\
\hline 25 & 27.63 & $0.17 \pm 0.01$ & 26.84 & $0.30 \pm 0.02$ \\
\hline 26 & 28.36 & $0.55 \pm 0.01$ & 27.47 & $0.94 \pm 0.01$ \\
\hline 27 & 28.84 & $1.65 \pm 0.01$ & 28.06 & $2.94 \pm 0.02$ \\
\hline $\begin{array}{l}28 \\
29\end{array}$ & 30.02 & $0.82 \pm 0.02$ & 29.02 & $2.40 \pm 0.01$ \\
\hline 30 & 31.19 & $0.08 \pm 0.01$ & 30.10 & $0.17 \pm 0.01$ \\
\hline 31 & 32.61 & $1.18 \pm 0.01$ & 31.56 & $4.93 \pm 0.01$ \\
\hline 32 & 33.26 & $0.12 \pm 0.02$ & 32.53 & $0.24 \pm 0.01$ \\
\hline $\begin{array}{l}33 \\
34\end{array}$ & 34.58 & $0.34 \pm 0.01$ & 33.47 & $0.81 \pm 0.01$ \\
\hline 35 & 36.19 & $0.15 \pm 0.01$ & 35.28 & $0.27 \pm 0.01$ \\
\hline 36 & 37.53 & $0.23 \pm 0.01$ & 36.73 & $0.68 \pm 0.02$ \\
\hline 37 & 38.85 & $0.18 \pm 0.01$ & 38.01 & $0.34 \pm 0.01$ \\
\hline $\begin{array}{l}38 \\
39\end{array}$ & 39.79 & $0.64 \pm 0.01$ & 38.97 & $2.06 \pm 0.02$ \\
\hline 40 & ND & ND & 42.49 & $0.28 \pm 0.01$ \\
\hline 41 & ND & ND & 42.80 & $0.17 \pm 0.01$ \\
\hline 42 & ND & ND & 44.254 & $0.23 \pm 0.01$ \\
\hline 43 & ND & ND & 45.39 & $0.37 \pm 0.01$ \\
\hline 44 & 46.93 & $0.32 \pm 0.02$ & 46.21 & $1.09 \pm 0.03$ \\
\hline 45 & 47.81 & $0.17 \pm 0.05$ & 47.07 & $0.92 \pm 0.02$ \\
\hline 46 & 48.94 & $0.18 \pm 0.01$ & 48.30 & $0.40 \pm 0.01$ \\
\hline 47 & 54.09 & $0.12 \pm 0.01$ & 53.30 & $0.34 \pm 0.02$ \\
\hline 48 & ND & ND & 54.21 & $0.31 \pm 0.01$ \\
\hline 49 & 56.21 & $0.27 \pm 0.01$ & 55.57 & $0.93 \pm 0.01$ \\
\hline 50 & ND & ND & 56.68 & $0.38 \pm 0.01$ \\
\hline 51 & 58.82 & $0.35 \pm 0.01$ & 58.29 & $1.28 \pm 0.01$ \\
\hline Sum & & $19.57 \pm 0.05$ & & $41.68 \pm 0.09$ \\
\hline
\end{tabular}

ND: Not detected. 


\subsection{Comparison between Vanillin-Sulfuric Acid Assay and UPLC-PDA}

Considering the vanillin-sulfuric acid assay is a classical and popular spectrophotometric method for quantifying saponins from various plant materials [18], the developed UPLC-PDA method was compared with vanillin-sulfuric acid assay in the present work. C. oleifera seed saponins have been determined by this colorimetric method, and the detection wavelength was set at $538 \mathrm{~nm}[19,20]$. However, the maximum absorption of reaction mixtures for theasaponin $\mathrm{E}_{1}, 70 \%$ methanol extract and the total saponin fraction of $C$. sinensis seeds were found at $600 \mathrm{~nm}$ (Figure 3). The standard curve for theasaponin $E_{1}$ was $Y=1.166 X-0.1221\left(R^{2}=0.9982\right)$, where $X$ was the concentration and $Y$ was the absorbance. The $95 \%$ confidence intervals for the slope and y-intercept were estimated to be 1.051 to 1.271 , and -0.190 to -0.047 . The contents of saponins in the $70 \%$ methanol extract and the purified total saponin fraction were determined to be $43.11 \pm 3.17 \%$ (wt \%) and $56.60 \pm 5.79 \%$ (wt \%), respectively. These results were significantly higher than those determined by the UPLC-PDA method $(p<0.05)$. The former value was more than twice, and the latter was 1.36 folds as compared with the UPLC-PDA data $(p<0.05)$.

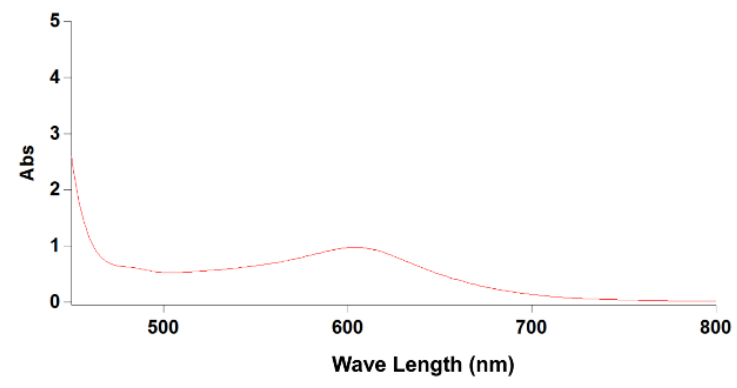

(A)

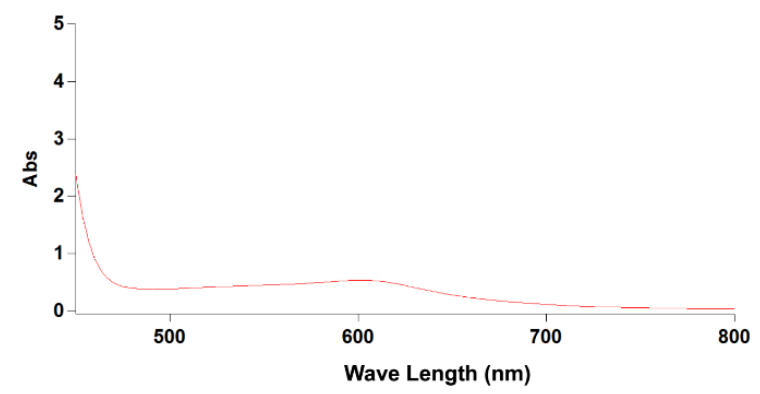

(B)

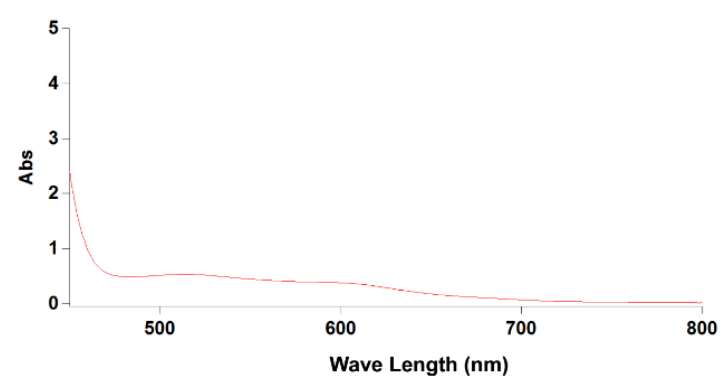

(C)

Figure 3. UV-Vis spectra of reaction mixture from vanillin-sulfuric acid assay between $450 \mathrm{~nm}$ and $800 \mathrm{~nm}$. (A) Theasaponin $E_{1}(1.0 \mathrm{mg} / \mathrm{mL})$. (B) Total saponin fraction of C. sinensis seeds (1.0 mg/mL).

(C) Crude extract of C. sinensis seeds $(1.0 \mathrm{mg} / \mathrm{mL})$.

Differences in selection of reagent, condition of full color development, standard, and wavelength make it hard to compare the results from different studies using this spectrophotometric assay [18]. More importantly, the vanillin-acid reagent could give a color reaction under acidic conditions with tannins [42], flavonoids [43], proanthocyanidins [44], and steroids [45], thus provide nonspecific and misleading information [21]. As shown in Figure 3, the absorption peaks of the total saponin fraction and crude extract were not obvious, indicating the absorption value might be easily interfered by other impurities. Although several trace saponins were not detected in the crude extract and purified saponin fraction $(2 \mathrm{mg} / \mathrm{mL})$ by UPLC-PDA, we thought that the exorbitant detection result from vanillin-sulfuric acid was mainly caused by some interfering substances such as flavonoids. Given the convenience and low cost, the vanillin-sulfuric acid assay could be used to quantify purified $C$. sinensis 
seed saponins, and give the comparable result with the UPLC-PDA method. However, it is not suitable for determining the saponin content in the crude extract of $C$. sinensis seeds.

\section{Materials and Methods}

\subsection{Plant Materials and Chemicals}

The full ripe fruits of tea (Camellia sinensis (L.) O. Kuntze, Theaceae) variety Fudingdabai were harvested from the Tea Resource Garden of China National Tea Museum (Hangzhou, China). The color of these fruits was brown, and their diameter was between $2.7 \mathrm{~cm}$ and $3.3 \mathrm{~cm}$. Methanol and acetonitrile of HPLC grade were purchased from Tianjin Shield Company (Tianjin, China). Ethanol, methanol, formic acid, and vanillin of analytical grade were obtained from the National Medicine Chemical Reagent Co. Ltd. (Shanghai, China). The ultrapure water was prepared by a Milli-Q water purification system (Millipore, Bedford, MA, USA).

\subsection{Preparation of Tea Seed Extract, Total Saponin Fraction and Standard Substance (Theasaponin $E_{1}$ )}

Tea seeds were removed from shells, freeze-dried and milled into powder by a pulverizer. Then, $800 \mathrm{~g}$ powder was extracted under reflux with $12 \mathrm{~L}$ of $70 \%$ methanol aqueous solution at $70{ }^{\circ} \mathrm{C}$ for $5 \mathrm{~h}$. The filtered decoction was concentrated by rotary evaporation, and then lyophilized by a freeze dryer (LGJ-10C, Beijing Sihuan Science Instrument Factory, Bejing, China). The yield of extract from tea seeds was $106.06 \mathrm{~g}(13.26 \%)$.

The crude tea seed extract was suspended in water and extracted successively with petroleum ether, ethyl acetate (EtOAc), and 1-butanol $(n-\mathrm{BuOH})$ thrice, respectively. The $n$-BuOH fraction was concentrated under reduced pressure, subjected to D101 column chromatography, and then eluted consecutively with $\mathrm{H}_{2} \mathrm{O}, 30 \%, 50 \%$, and $70 \%$ ethanol-aqueous solution $(v / \mathrm{v})$ at the flow rate of $3 \mathrm{~mL} / \mathrm{min}$ each for 2 bed volume (BV, $2.5 \mathrm{~L}$ ). The 30\%, 50\%, and 70\% ethanol-eluted fractions were collected, concentrated, and lyophilized to yield $9.04 \mathrm{~g}, 24.69 \mathrm{~g}$ and $3.09 \mathrm{~g}$, respectively. Each fraction was detected by the UPLC-PDA-MS method as described in the Section 2.3 . The $50 \%$ ethanol-eluted fraction had the similar saponin compositions with the crude tea seed extract, and was used as the total saponin fraction for the next work.

Theasaponin $\mathrm{E}_{1}$ (21-O-angeloyl-22-O-acetyltheasapogenol E 3-O- $\beta$-D-galactopyranosyl- $(1 \rightarrow 2)$ [ $\beta$-D-xylopyranosyl-( $1 \rightarrow 2)-\alpha$-L-arabinopyranosyl- $(1 \rightarrow 3)]-\beta$-D-glucopyranosiduronic acid) was prepared using a method developed in our lab. Briefly, the total saponin fraction was purified by reversed-phase preparative HPLC system (GE ÄKTA purifier100, Uppsala, Sweden) equipped with a SinoChrom ODS-BP column $(5 \mu \mathrm{m}, 250 \mathrm{~mm} \times 10.0 \mathrm{~mm}$ i.d., Elite, Dalian, China). Elution was performed with acetonitrile/ $\mathrm{H}_{2} \mathrm{O} /$ formic acid (50.0:49.9:0.1, v/v/v) at the wavelength of $210 \mathrm{~nm}$ and a flow rate of $2.0 \mathrm{~mL} / \mathrm{min}$ to afford three fractions. The second fraction was further purified on a Waters XBridge Shield RP18 column (5 $\mu \mathrm{m}, 250 \mathrm{~mm} \times 10.0 \mathrm{~mm}$ i.d., Waters, Milford, MA, USA) to yield theasaponin E1. The mobile phase A was formic acid/water $(0.1: 99.9, v / v)$, and B was formic acid/acetonitrile $(0.1: 99.9, v / v)$. The elution gradient was $38 \%$ B from 0 to $15 \mathrm{~min}$, then increased to $40 \% \mathrm{~B}$ and maintained for $35 \mathrm{~min}$. The flow rate of the mobile phase was kept at $1.5 \mathrm{~mL} / \mathrm{min}$. The purity of the isolated theasaponin $\mathrm{E}_{1}$ was determined to be $98 \%$ based on the percentage of peak area detected by UPLC-PDA-MS (Figure A1). ${ }^{1} \mathrm{H}$ - and ${ }^{13} \mathrm{C}-\mathrm{NMR}$ spectra were recorded on a Bruker AV-400 (Karlsruhe, Germany) at 500 and $125 \mathrm{MHz}$, respectively (Table A1). The spectral data (MS, ${ }^{1} \mathrm{H}-$ and $\left.{ }^{13} \mathrm{C}-\mathrm{NMR}\right)$ of the obtained theasaponin $\mathrm{E}_{1}$ were consistent with the published data [26].

\subsection{Separation and Identification of Saponins by UPLC-PDA-QTOF-MS/MS}

The chromatographic separation was performed on an Acquity UPLC instrument equipped with a PDA detector (Waters, Milford, MA, USA) and a Waters Acquity UPLC HSS T3 column (1.8 $\mu \mathrm{m}$, $150 \mathrm{~mm} \times 2.1 \mathrm{~mm}$ i.d., Waters, Milford, MA, USA). The PDA detector was set to scan a range from $200 \mathrm{~nm}$ to $400 \mathrm{~nm}$, and the specific wavelength of $210 \mathrm{~nm}$ was selected for detecting saponins. The 
mobile phase A was water containing $0.1 \%$ formic acid, and B was acetonitrile containing $0.1 \%$ formic acid. Gradient elution was performed as follows: 0-4 min, 35-37\% B; 4-32 min, 37\% B; 32-58 min, $37-45 \%$ B; 58-60 min, 35\% B. The sample injection volume was $5 \mu \mathrm{L}$, the column was kept at $30{ }^{\circ} \mathrm{C}$, and the flow rate was $0.2 \mathrm{~mL} / \mathrm{min}$.

The mass spectrometry analysis was carried out using a hybrid triple quadrupole time-of-flight mass spectrometer (Triple TOFTM 5600 plus System, AB SCIEX, Foster City, CA, USA) equipped with an electrospray ionization (ESI) source in negative ionization mode. The mass range was set at $m / z$ 100-2000 for both Time-of-Flight (TOF)-MS and TOF-MS/MS scan. Nitrogen was used as the nebulizer and auxiliary gas. The optimized MS conditions were as follows: ion spray voltage, $-4.5 \mathrm{kV}$; ion source temperature, $500^{\circ} \mathrm{C}$; nebulizing gas (Gas 1), 50 psi; Tis gas (Gas 2), 50 psi; curtain gas, 35 psi; declustering potential (DP), $100 \mathrm{~V}$; collision energy (CE), $10 \mathrm{~V}$. For the MS/MS acquisition mode, the sweeping collision energy was set at $60 \pm 20 \mathrm{~V}$ for collision-induced dissociation (CID). Data acquisition was carried out using Peak View Software 1.2.0.3 software (AB Sciex, Foster City, CA, USA) in IDA (information-dependent acquisition) mode.

\subsection{Quantification of Saponins by UPLC-PDA}

Quantitative analysis of the individual saponins in the tea seeds was conducted using UPLC-PDA as described in Section 2.3. The method was validated according to Guidance for Industry Bioanalytical Method Validation (FDA) [27]. Theasaponin $\mathrm{E}_{1}$ was used as a reference standard, and the calibration curve was constructed at seven concentrations in the range of 50-1000 $\mu \mathrm{g} / \mathrm{mL}$. The "intra-day" and "inter-day" accuracy and precision of the method were determined with three concentration levels $(60,100$, and $500 \mu \mathrm{g} / \mathrm{mL})$ of theasaponin $\mathrm{E}_{1}$ on the same day (five replicates) or on five different days. The accuracy, expressed as the percent deviation (\% DEV), was calculated as (mean measured concentration - nominal concentration/nominal concentration) $\times 100 \%$. The precision, expressed as the relative standard deviation (RSD), was calculated as (standard deviation/mean measured concentration) $\times 100 \%$ The lower limits of detection (LLOD) and quantification (LLOQ) were defined as analyte concentrations with a signal-to-noise $(\mathrm{S} / \mathrm{N})$ ratio of 3 and 10 , respectively, by injecting a series of dilute solutions with known concentrations.

\subsection{Vanillin-Sulfuric Acid Assay}

The total saponin content of tea seeds was determined following the procedures described by Chen et al. with a few modifications [19]. Briefly, $0.5 \mathrm{~mL}$ of sample solution was sequentially reacted with $0.5 \mathrm{~mL}$ of vanillin ethanol solution $(8 \%, w / v)$ and $4 \mathrm{~mL}$ of diluted sulfuric acid $(72 \%, w / v)$. After incubation at $60{ }^{\circ} \mathrm{C}$ for $10 \mathrm{~min}$, the reaction mixture was cooled in an ice water bath for another $15 \mathrm{~min}$, and scanned from 450 to $800 \mathrm{~nm}$ by an UV-VIS spectrophotometer (UV-2550, Shimadzu, Japan). Theasaponin $\mathrm{E}_{1}(0.2-1.0 \mathrm{mg} / \mathrm{mL})$ was used as a reference standard to establish the calibration curve.

\subsection{Statistical Analysis}

The data were presented as the mean \pm standard deviation (SD). Statistical differences between the two sets of data were evaluated using the Student's t-test, and a $p$ value $<0.05$ was considered statistically significant. All statistical analysis of data was performed using the SPSS (Statistical Product and Service Solutions) statistics [26].

\section{Conclusions}

A novel UPLC-PDA-QTOF-MS/MS method was developed and validated for tentatively determining 51 triterpene saponins in $C$. sinensis seeds. Based on the retention behavior, fragmentation pattern and previous literatures, six potentially new saponins $(1,6,38,42,46,44$, or 47) were detected. One saponin (36) identified as camelliasaponin $\mathrm{A}_{1}$ or $\mathrm{A}_{2}$ from the $C$. japonica seeds [34] was found in the $C$. sinensis seeds for the first time. Compared with the vanillin-sulfuric acid assay, the UPLC-PDA method could quantify individual saponins, and provide more reliable quantitative test results for the 
total saponin content. Its linearity, accuracy and precision were satisfactory under the requirements of the Guidance for Industry: Bioanalytical Method Validation (FDA, 2013). This work offered a possible routine method for the identification and quantification of $C$. sinensis seed saponins, which was of great significance for the future investigations and applications of these compounds. Moreover, this analytical method could be further developed and validated for the quantification of the saponins in different organs of $C$. sinensis and other Camellia species such as C. reticulata, C. japonica, and C. sasanqua, etc.

Author Contributions: Optimization of UPLC-PDA-QTOF-MS chromatography and data analysis, X.W. and B.L.; preparation of extract and total saponin fraction, L.J.; vanillin-sulfuric acid assay, J.W. and Y.L.; validation, H.K., X.L., and P.L; writing-review and editing, B.L.; supervision, P.H. and Y.T.

Funding: This research was funded by the Key Research and Development Program of Zhejiang Province (grant no. 2018C02012), the National Key Research and Development Program of China (grant no. 2017YFD0400800), the National Natural Science Foundation of China (grant no. 31501474), the Natural Science Foundation of Zhejiang Province (grant no. LY15C200007), the Fundamental Research Funds for the Central Universities, Fujian Province "2011 Collaborative Innovation Center" of Chinese Oolong Tea Project (Fujian Educational Department, (2015) No.75), and the 2016 Project of Funding Municipal Project.

Acknowledgments: This research was assisted by Analysis Center of Agrobiology and Environmental Sciences \& Institute of Agrobiology and Environmental Sciences, Zhejiang University.

Conflicts of Interest: The authors declare no conflict of interest.

\section{Appendix A}

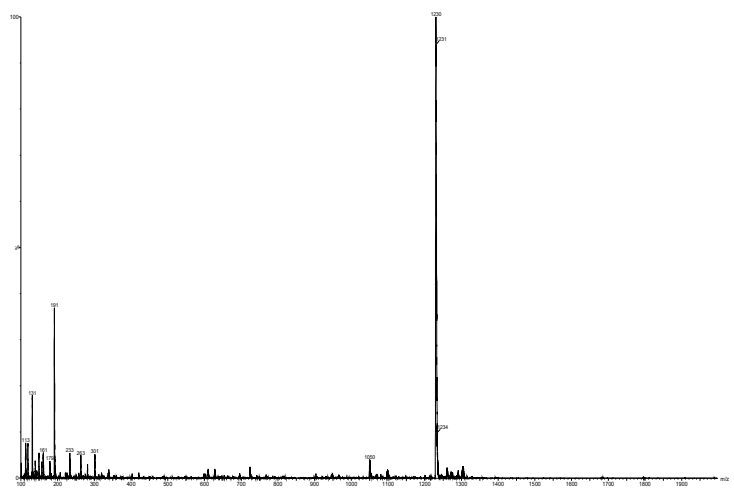

(A)

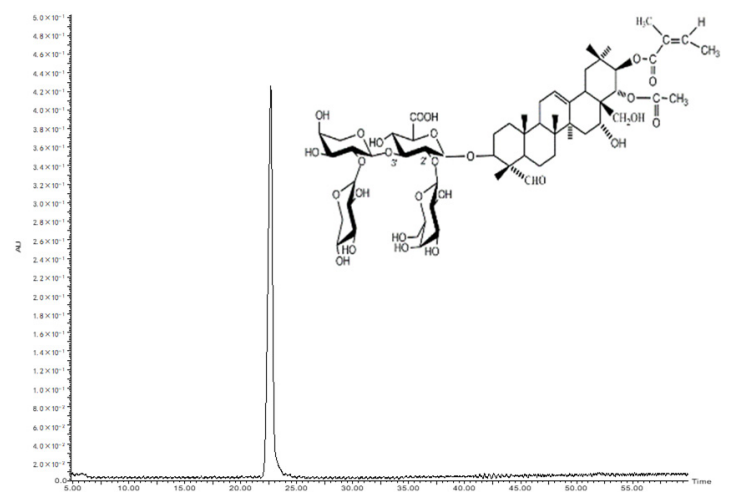

(B)

Figure A1. UPLC-PDA-MS chromatogram of theasaponin $E_{1}$ prepared in this work. (A) UPLC-UV chromatogram detected at $210 \mathrm{~nm}$ and chemical structure of theasaponin $\mathrm{E}_{1}$. (B) MS spectra of theasaponin $\mathrm{E}_{1}$ in negative ion mode.

Table A1. ${ }^{13} \mathrm{C}$ - and ${ }^{1} \mathrm{H}-\mathrm{NMR}$ spectral data of theasaponin $\mathrm{E}_{1}$ prepared in this work (pyridine-d5).

\begin{tabular}{ccc}
\hline Carbon & ${ }^{13}$ C-NMR $\delta(\mathbf{p p m})$ & ${ }^{\mathbf{1}} \mathbf{H}-\mathbf{N M R} \boldsymbol{\delta}(\mathbf{p p m})$, Multiplicity, $\mathbf{J}_{\mathbf{H H}} \mathbf{( H z )}$ \\
\hline C-1 & 37.91 & \\
C-2 & 25.00 \\
C-3 & 84.28 \\
C-4 & 54.91 \\
C-5 & 48.08 \\
C-6 & 20.12 \\
C-7 & 32.15 \\
C-8 & 40.03 \\
C-9 & 46.51 \\
C-10 & 35.78 \\
C-11 & 23.52 \\
\hline
\end{tabular}


Table A1. Cont.

\begin{tabular}{|c|c|c|}
\hline Carbon & ${ }^{13} \mathrm{C}-\mathrm{NMR} \delta$ (ppm) & ${ }^{1} \mathrm{H}-\mathrm{NMR} \delta(\mathrm{ppm})$, Multiplicity, $J_{H H}(\mathrm{~Hz})$ \\
\hline $\mathrm{C}-12$ & 122.75 & $5.38(1 \mathrm{H})$, br s \\
\hline C-13 & 142.67 & \\
\hline C-14 & 41.44 & \\
\hline C-15 & 34.30 & \\
\hline C-16 & 67.67 & \\
\hline C-17 & 47.75 & \\
\hline $\mathrm{C}-18$ & 39.84 & \\
\hline C-19 & 46.90 & \\
\hline C-20 & 36.02 & \\
\hline $\mathrm{C}-21$ & 78.63 & $6.62(1 \mathrm{H}), \mathrm{d}, 10.1$ \\
\hline C-22 & 74.11 & $6.21(1 \mathrm{H}), \mathrm{d}, 10.1$ \\
\hline C-23 & 209.74 & $9.93(1 \mathrm{H}), \mathrm{s}$ \\
\hline C-24 & 10.82 & $1.48(3 \mathrm{H}), \mathrm{s}$ \\
\hline C-25 & 15.53 & $0.81(3 \mathrm{H}), \mathrm{s}$ \\
\hline$C-26$ & 16.54 & $0.87(3 \mathrm{H}), \mathrm{s}$ \\
\hline $\mathrm{C}-27$ & 27.14 & $1.78(3 \mathrm{H}), \mathrm{s}$ \\
\hline C-28 & 63.57 & \\
\hline C-29 & 29.22 & $1.10(3 \mathrm{H}), \mathrm{s}$ \\
\hline$C-30$ & 20.02 & $1.22(3 \mathrm{H}), \mathrm{s}$ \\
\hline GlcA- $1^{\prime}$ & 103.91 & $4.86(1 \mathrm{H}), \mathrm{d}, 7.3$ \\
\hline $2^{\prime}$ & 78.02 & \\
\hline $3^{\prime}$ & 83.78 & \\
\hline $4^{\prime}$ & 70.58 & \\
\hline $5^{\prime}$ & 77.04 & \\
\hline $6^{\prime}$ & 171.89 & \\
\hline Gal-1"1 & 103.02 & $5.79(1 \mathrm{H}), \mathrm{d}, 7.6$ \\
\hline $2^{\prime \prime}$ & 73.47 & \\
\hline $3^{\prime \prime}$ & 75.15 & \\
\hline $4^{\prime \prime}$ & 70.27 & \\
\hline $5^{\prime \prime}$ & 76.32 & \\
\hline $6^{\prime \prime}$ & 61.82 & \\
\hline Ara- $1^{\prime \prime \prime}$ & 101.39 & $5.77(1 \mathrm{H}), \mathrm{d}, 6.5$ \\
\hline $2^{\prime \prime \prime}$ & 82.08 & \\
\hline $3^{\prime \prime \prime}$ & 73.12 & \\
\hline $4^{\prime \prime \prime}$ & 68.09 & \\
\hline $5^{\prime \prime \prime}$ & 65.75 & \\
\hline 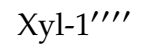 & 106.82 & $5.02(1 \mathrm{H}), \mathrm{d}, 7.6$ \\
\hline $2^{\prime \prime \prime \prime}$ & 75.70 & \\
\hline $3^{\prime \prime \prime \prime}$ & 77.99 & \\
\hline $4^{\prime \prime \prime \prime}$ & 70.51 & \\
\hline $5^{\prime \prime \prime \prime}$ & 67.27 & \\
\hline Ang- $1^{\prime \prime \prime \prime \prime \prime}$ & 167.61 & \\
\hline $2^{\prime \prime \prime \prime \prime}$ & 128.73 & \\
\hline $3^{\prime \prime \prime \prime \prime}$ & 136.78 & $6.00(1 \mathrm{H})$, dq-like \\
\hline $4^{\prime \prime \prime \prime \prime}$ & 15.65 & $2.11(3 \mathrm{H}), \mathrm{d}, 7.0$ \\
\hline $5^{\prime \prime \prime \prime \prime}$ & 20.76 & $2.03(3 \mathrm{H}), \mathrm{s}$ \\
\hline Ac- $1^{\prime \prime \prime \prime \prime \prime}$ & 170.72 & \\
\hline $2^{\prime \prime \prime \prime \prime \prime}$ & 20.62 & $1.93(3 \mathrm{H}), \mathrm{s}$ \\
\hline
\end{tabular}

GlcA: $\beta$-D-glucopyranosiduronic acid; Gal: $\beta$-D-galactopyranosyl; $\quad$ Ara: $\alpha$-L-arabinopyranosyl; Xyl: $\beta$-D-xylopyranosyl; Glc: $\beta$-D-glucopyranosyl; Ac: acetyl; Ang: angeloyl.

\section{References}

1. Mukhopadhyay, M.; Mondal, T.K.; Chand, P.K. Biotechnological advances in tea (Camellia sinensis [L.] O. Kuntze): A review. Plant Cell Rep. 2016, 35, 255-287. [CrossRef] [PubMed] 
2. Li, B.; Vik, S.B.; Tu, Y.Y. Theaflavins inhibit the ATP synthase and the respiratory chain without increasing superoxide production. J. Nutr. Biochem. 2012, 23, 953-960. [CrossRef] [PubMed]

3. Demirbas, A. Tea seed upgrading facilities and economic assessment of biodiesel production from tea seed oil. Energy Convers. Manage. 2010, 51, 2595-2599. [CrossRef]

4. Li, N.; Morikawa, T.; Matsuda, H.; Ninomiya, K.; Li, X.A.; Yoshikawa, M. New flavanone oligoglycosides, theaflavanosides I, II, III, and IV, with hepatoprotective activity from the seeds of tea plant (Camellia sinensis). Heterocycles 2007, 71, 1193-1201.

5. Li, B.; Xu, Y.; Jin, Y.; Wu, Y.; Tu, Y. Response surface optimization of supercritical fluid extraction of kaempferol glycosides from tea seed cake. Ind. Crop. Prod. 2010, 32, 123-128. [CrossRef]

6. Shao, P.; Zhu, Y.Q.; Liu, Q.; Zhang, T.T.; Sun, P.L. Isolation, antioxidant and antitumor in vitro activities of cold pressed green tea seed cake polysaccharide. Curr. Top Nutraceut. R 2015, 13, 189-196.

7. Zhao, P.; Gao, D.F.; Xu, M.; Shi, Z.G.; Wang, D.; Yang, C.R.; Zhang, Y.J. Triterpenoid saponins from the genus Camellia. Chem. Biodivers. 2011, 8, 1931-1942. [CrossRef]

8. Guo, N.; Tong, T.T.; Ren, N.; Tu, Y.Y.; Li, B. Saponins from seeds of Genus Camellia: Phytochemistry and bioactivity. Phytochemistry 2018, 149, 42-55. [CrossRef]

9. Murakami, T.; Nakamura, J.; Matsuda, H.; Yoshikawa, M. Bioactive saponins and glycosides. XV. Saponin constituents with gastroprotective effect from the seeds of tea plant, Camellia sinensis L. var. assamica Pierre, cultivated in Sri Lanka: Structures of assamsaponins A, B, C, D, and E. Chem. Pharm. Bull 1999, 47, 1759-1764. [CrossRef]

10. Murakami, T.; Nakamura, J.; Kageura, T.; Matsuda, H.; Yoshikawa, M. Bioactive saponins and glycosides. XVII. Inhibitory effect on gastric emptying and accelerating effect on gastrointestinal transit of tea saponins: Structures of assamsaponins F, G, H, I, and J from the seeds and leaves of the tea plant. Chem Pharm Bull. 2000, 48, 1720-1725. [CrossRef]

11. Jia, L.Y.; Wu, X.J.; Gao, Y.; Rankin, G.O.; Pigliacampi, A.; Bucur, H.; Li, B.; Tu, Y.Y.; Chen, Y.C. Inhibitory effects of total triterpenoid saponins isolated from the seeds of the tea plant (Camellia sinensis) on human ovarian cancer cells. Molecules 2017, 22, 1649. [CrossRef] [PubMed]

12. Li, N.; Ma, Z.J.; Chu, Y.; Wang, Y.; Li, X. Phytochemical analysis of the triterpenoids with cytotoxicity and QR inducing properties from the total tea seed saponin of Camellia sinensis. Fitoterapia 2013, 84, 321-325. [CrossRef] [PubMed]

13. Yang, W.S.; Ko, J.; Kim, E.; Kim, J.H.; Park, J.G.; Sung, N.Y.; Kim, H.G.; Yang, S.; Rho, H.S.; Hong, Y.D.; et al. 21-O-Angeloyltheasapogenol E3, a Novel triterpenoid saponin from the seeds of tea plants, inhibits macrophage-mediated inflammatory responses in a NF-kappa B-dependent manner. Mediators Inflamm. 2014, 2014, 658351. [CrossRef] [PubMed]

14. Boran, H.; Ciftci, C.; Er, A.; Kose, O.; Kurtoglu, I.Z.; Kayis, S. Evaluation of antibacterial activity of green tea (Camellia sinensis L.) seeds against some fish pathogens in rainbow trout (Oncorhynchus mykiss, Walbaum). Turk. J. Fish. Aquat. Sci. 2015, 15, 49-57.

15. Joshi, R.; Sood, S.; Dogra, P.; Mahendru, M.; Kumar, D.; Bhangalia, S.; Pal, H.C.; Kumar, N.; Bhushan, S.; Gulati, A.; et al. In vitro cytotoxicity, antimicrobial, and metal-chelating activity of triterpene saponins from tea seed grown in Kangra valley, India. Med. Chem. Res. 2013, 22, 4030-4038. [CrossRef]

16. Kim, J.D.; Khan, M.I.; Shin, J.H.; Lee, M.G.; Seo, H.J.; Shin, T.S.; Kim, M.Y. HPLC fractionation and pharmacological assessment of green tea seed saponins for antimicrobial, anti-angiogenic and hemolytic activities. Biotechnol. Bioprocess Eng. 2015, 20, 1035-1043. [CrossRef]

17. Cay, S. Enhancement of cadmium uptake by Amaranthus caudatus, an ornamental plant, using tea saponin. Environ. Monit Assess. 2016, 188, 320. [CrossRef]

18. Cheok, C.Y.; Salman, H.A.K.; Sulaiman, R. Extraction and quantification of saponins: A review. Food Res. Int. 2014, 59, 16-40. [CrossRef]

19. Chen, Y.F.; Yang, C.H.; Chang, M.S.; Ciou, Y.P.; Huang, Y.C. Foam properties and detergent abilities of the saponins from Camellia oleifera. Int. J. Mol. Sci. 2010, 11, 4417-4425. [CrossRef]

20. Li, T.T.; Zhang, H.; Wu, C.E. Screening of antioxidant and antitumor activities of major ingredients from defatted Camellia oleifera seeds. Food Sci. Biotechnol. 2014, 23, 873-880. [CrossRef]

21. Oleszek, W.A. Chromatographic determination of plant saponins. J. Chromatogr. A 2002, 967, 147-162. [CrossRef] 
22. Oleszek, W.; Bialy, Z. Chromatographic determination of plant saponins-An update (2002-2005). J. Chromatogr. A. 2006, 1112, 78-91. [CrossRef] [PubMed]

23. Cao, D.; Wang, Q.; Jin, J.; Qiu, M.S.; Zhou, L.; Zhou, X.H.; Li, H.; Zhao, ZX. Simultaneous qualitative and quantitative analyses of triterpenoids in Ilex pubescens by ultra-high-performance liquid chromatography coupled with quadrupole time-of-flight mass spectrometry. Phytochem. Anal. 2018, 29, 168-179. [CrossRef] [PubMed]

24. Foubert, K.; Cuyckens, F.; Vleeschouwer, K.; Theunis, M.; Vlietinck, A.; Pieters, L.; Apers, S. Rapid quantification of 14 saponins of Maesa lanceolata by UPLC-MS/MS. Talanta 2010, 81, 1258-1263. [CrossRef]

25. Wang, Y.; Xu, R.C.; Xiao, J.; Zhang, J.; Wang, X.H.; An, R.; Ma, Y.M. Quantitative analysis of flavonoids, alkaloids and saponins of Banxia Xiexin decoction using ultra-high performance liquid chromatography coupled with electrospray ionization tandem mass spectrometry. J. Pharmaceut. Biomed. 2014, 88, 525-535. [CrossRef]

26. Kitagawa, I.; Hori, K.; Motozawa, T.; Murakami, T.; Yoshikawa, M. Structures of new acylated oleanene-type triterpene oligoglycosides, theasaponins $\mathrm{E}_{1}$ and $\mathrm{E}_{2}$, from the seeds of tea plant, Camellia sinensis (L.) O. Kuntze. Chem. Pharm. Bull 1998, 46, 1901-1906. [CrossRef]

27. U.S. Department of Health and Human Service, Food and Drug Administration, Center for Drug Evaluation and Research, Center for Veterinary Medicine. Bioanalytical Method Validation Guidance for Industry; Center for Drug Evaluation and Research: Silver Spring, MD, USA, 2018.

28. Avula, B.; Wang, Y.H.; Rumalla, C.S.; Ali, Z.; Smillie, T.J.; Khan, I.A. Analytical methods for determination of magnoflorine and saponins from roots of Caulophyllum thalictroides (L.) Michx. Using UPLC, HPLC and HPTLC. J. Pharmaceut. Biomed. 2011, 56, 895-903. [CrossRef]

29. Yoshikawa, M.; Morikawa, T.; Nakamura, S.; Li, N.; Li, X.; Matsuda, H. Bioactive saponins and glycosides. XXV. Acylated oleanane-type triterpene saponins from the seeds of tea plant (Camellia sinensis). Chem. Pharm. Bull. 2007, 55, 57-63. [CrossRef]

30. Morikawa, T.; Li, N.; Nagatomo, A.; Matsuda, H.; Li, X.; Yoshikawa, M. Triterpene saponins with gastroprotective effects from tea seed (the seeds of Camellia sinensis). J. Nat. Prod. 2006, 69, 185-190. [CrossRef]

31. Morikawa, T.; Matsuda, H.; Li, N.; Li, X.; Yoshikawa, M. Bioactive Saponins and glycosides. Part 29 Acylated oleanane-type triterpene saponins: Theasaponins $\mathrm{A}_{6}, \mathrm{~A}_{7}$, and $\mathrm{B}_{5}$, from the seeds of Camellia sinensis. Helv. Chim. Acta 2007, 90, 2342-2348. [CrossRef]

32. Myose, M.; Warashina, T.; Miyase, T. Triterpene saponins with hyaluronidase inhibitory activity from the seeds of Camellia sinensis. Chem. Pharm. Bull. 2012, 60, 612-623. [CrossRef] [PubMed]

33. Yoshikawa, M.; Morikawa, T.; Li, N.; Nagatomo, A.; Li, X.; Matsuda, H. Bioactive saponins and glycosides. XXIII. Triterpene saponins with gastroprotective effect from the seeds of Camellia sinensis - Theasaponins $\mathrm{E}_{3}$, $\mathrm{E}_{4}, \mathrm{E}_{5}, \mathrm{E}_{6}$, and $\mathrm{E}_{7}$. Chem. Pharm. Bull 2005, 53, 1559-1564. [CrossRef] [PubMed]

34. Yoshikawa, M.; Murakami, T.; Yoshizumi, S.; Murakami, N.; Yamahara, J.; Matsuda, H. Bioactive saponins and glycosides.5. Acylated polyhydroxyolean-12-ene triterpene oligoglycosides, camelliasaponins $\mathrm{A}_{1}, \mathrm{~A}_{2}, \mathrm{~B}_{1}$, $\mathrm{B}_{2}, \mathrm{C}_{1}$, and $\mathrm{C}_{2}$, from the seeds of Camellia japonica L: Structures and inhibitory activity on alcohol absorption. Chem. Pharm. Bull. 1996, 44, 1899-1907. [CrossRef] [PubMed]

35. Li, N.; Li, X.; Feng, Z. Chemical constituents of tea seed saponins part from Camellia sinensis cultivated in China. J. Shenyang Pharm. Univ. 2008, 25, 544-548.

36. Morikawa, T.; Matsuda, H.; Li, N.; Nakamura, S.; Li, X.; Yoshikawa, M. Bioactive saponins and glycosides. XXVI. New triterpene saponins, theasaponins E10, E11, E12, E13, and G2, from the seeds of tea plant (Camellia sinensis). Heterocycles 2006, 68, 1139.

37. Fu, H.Z.; Wan, K.H.; Yan, Q.W.; Zhou, G.P.; Feng, T.T.; Dai, M.; Zhong, R.J. Cytotoxic triterpenoid saponins from the defatted seeds of Camellia oleifera Abel. J. Asian Nat. Prod. Res. 2018, 20, 412-422. [CrossRef]

38. Han, S.Y.; Qiao, J.Q.; Zhang, Y.Y.; Yang, L.L.; Lian, H.Z.; Ge, X.; Chen, H.Y. Determination of n-octanol/water partition coefficient for DDT-related compounds by RP-HPLC with a novel dual-point retention time correction. Chemosphere 2011, 83, 131-136. [CrossRef]

39. Liang, C.; Qiao, J.Q.; Lian, H.Z. Determination of reversed-phase high performance liquid chromatography based octanol-water partition coefficients for neutral and ionizable compounds: Methodology evaluation. J. Chromatogr. A 2017, 1528, 25-34. [CrossRef] 
40. Poole, S.K.; Poole, C.F. Separation methods for estimating octanol-water partition coefficients. J. Chromatogr. B 2003, 797, 3-19. [CrossRef]

41. Schlotterbeck, G.; Ceccarelli, S.M. LC-SPE-NMR-MS: A total analysis system for bioanalysis. Bioanalysis 2009, 1, 549-559. [CrossRef]

42. Liu, M.X.; Wang, Y.W.; Han, J.G.; Mao, P.S. The content and distribution of condensed tannins in different species of the genus sorghum (Sorghum Moench) and their effect on seed protein electrophoresis. J. Sci. Food Agric. 2009, 89, 1446-1452. [CrossRef]

43. Uchimiya, M.; Ni, X.Z.; Wang, M. L Structure-reactivity relationships between the fluorescent chromophores and antioxidant activity of grain and sweet sorghum seeds. Food Sci. Nutr. 2016, 4, 811-817. [CrossRef] [PubMed]

44. Wang, Y.X.; McAllister, T.A.; Xu, Z.J.; Gruber, M.Y.; Skadhauge, B.; Jende-Strid, B.; Cheng, K.J. Effects of proanthocyanidins, dehulling and removal of pericarp on digestion of barley grain by ruminal micro-organisms. J. Sci. Food Agric. 1999, 79, 929-938. [CrossRef]

45. Matthews, J.S. Steroids. 223. Color reagent for steroids in thin-layer chromatography. Biochim. Biophys. Acta 1963, 69, 163-165. [CrossRef]

Sample Availability: Samples of the compounds are available from the authors. 